\title{
Influences of Morphology Parameters on the Contact Behavior of a Steel Interface
}

\author{
L. F. Fan*, L. Zhao* and X. M. Liu ${ }^{\dagger, \ddagger}$ \\ ${ }^{*}$ College of Architecture and Civil Engineering \\ Beijing University of Technology, Beijing 100124, P. R. China \\ ${ }^{\dagger}$ LNM, Institute of Mechanics \\ Chinese Academy of Sciences, Beijing 100124, P. R. China \\ ${ }^{\ddagger}$ xiaomingliu@imech.ac.cn
}

Received 14 October 2019

Revised 5 December 2019

Accepted 17 December 2019

Published 3 March 2020

\begin{abstract}
The surface roughness induced by geometric irregularities (asperities) has substantial influence on the contact stiffness, which further affects the working performance and service life of mechanical equipment. In this study, an elastic-plastic contact law between a sinusoidal asperity and a rigid smooth flat is first studied, which is then applied on a statistical model to simulate the contact behavior of a pair of $18 \mathrm{CrMo} 4$ steel surfaces to investigate the influences of morphology parameters on the contact stiffness. The analysis shows that smaller shape ratios $\xi$ and larger wavelengths $\lambda$ induce larger normal contact stiffness $K_{n}$ for surfaces with identical roughness, wherein the roughness is defined by the mean value of asperity heights $R_{a}$ and the standard deviation of asperity heights $R_{q}$. The normal contact stiffness increases as $R_{a}$ decreases under the same loading conditions, while the normal contact stiffness increases as $R_{q}$ decreases for surfaces with a fixed $R_{a}$. Besides, the normal pressure and normal contact stiffness derived from the proposed contact model are validated. The results demonstrate the potential of the proposed model in contact design due to its ability of establishing the relations between the normal contact stiffness and surface morphology parameters.
\end{abstract}

Keywords: Sinusoidal asperities; contact stiffness; elastic-plastic statistical model; 18CrMo4 steel interface; morphology parameters.

\section{Introduction}

Contact behaviors exist widely in mechanical-electrical and biomedical engineering [Du et al., 2011]. Contact stiffness is one of the important factors affecting the contact behavior, since it can substantially influence the overall static and dynamic characteristics of mechanical equipment, and thus the working performance of mechanical equipment.

¥Corresponding author. 
A large number of studies on contact stiffness have been reported, which can be classified into two groups: experimental studies and theoretical studies. The technology used in experiments mainly includes ultrasonic assessment Xiao and Sun, 2018], digital image correlation Kartal et al.. 2011]. model analysis [Boscolo and Banerjee, 2012] and virtual field methods [Giraudeau et al., 2010]. Theoretically, contact stiffness can be studied by Hertz theory Hertz. 1882], statistical theorv [Greenwood and Williamson, 1966] and fractal theory [Mandelbrot, 1983]. Numerical methods provide another efficient way to investigate the single asperity contact, which have been widely adopted in contact studies. Based on finite element model (FEM), the contact deformation of a sphere was divided into four distinct phases by Kogut and Etsion [2002] (i.e., the KE model). To further study the contact stiffness of a rough surface. FEM was combined with fractal theory and statistical theorv. Pan et al. 2017] established a fractal model based on 3D FEM, and the normal contact stiffness calculated by the model agreed well with experimental results. Under the condition of shoulder-shoulder contact between spheres, Zhao et al. 2015] proposed a statistical model for contact stiffness based on elastic-plastic FEM.

Many researchers [Saha et al., 2016; Saha and Jackson, 2016; Wang et al., 2017; Liu, 2014; Liu and Proudhon, 2014, 2016] have adopted sinusoidal (wavy) asperities in FEMs to study the contact behavior of rough surfaces since the sine function is foundational for Fourier series or Weierstrass-Mandelbrot function, which can be used to describe real surfaces in engineering Jackson and Streator. 2006: Wilson et al., 2010; Ciavarella et al., 2000]. Westergaard [1939] firstly obtained the elastic analytical solution for the contact between two slightly wavy surfaces in 1939. Gao et al. [2006] studied the effects of geometric and material parameters on plastic deformation and residual stress based on the contact between a wavy surface and a rigid flat surface. Johnson et al. [1985. established an elastic asymptotic solution (i.e., the JGH model) for the contact of a 3D sinusoidal surface. Saha et al. 2016] developed an elastic contact model under the condition of complete contact between a perfectly elastic 3D sinusoidal surface and a rigid flat surface. Saha and Jackson 2016] then extended it to an elastic-plastic contact model, and the results indicated that the deformation of a sinusoidal asperity was completely elastic when the amplitude was below a certain critical value. Moreover, Wang et al. [2017] also pointed out that a sinusoidal asperity was completely elastic when the amplitude was below a certain critical value under full stick contact conditions. Liu [2014] used FEM to study the effects of the geometrical parameters of sinusoidal asperities on the contact behavior under fully plastic deformation. Then, Liu and Proudhon [2014] divided the deformation of a sinusoidal asperity into elastic phase and plastic phase. For a more accurate description of the deformation evolution of a sinusoidal asperity, Liu and Proudhon [2016] further divided sinusoidal asperity deformation into five phases; however, the expression of critical deformation and the relation between deformation and force for each phase were not provided. 
The problem of sinusoidal and wavy surface contact has been studied for over 80 years. The shape ratio, defined as the ratio of asperity height to wavelength, was generally less than 0.40 Saha et al., 2016; Saha and Jackson, 2016; Liu, 2014; Liu and Proudhon, 2014, 2016; Gao et al., 2006]. However, the actual surfaces usually contain asperities with ratios greater than 0.40 , and the error affects the contact pressure [Liu, 2014; Liu and Proudhon, 2014]. Moreover, most of the previous reports concentrate on elastic and plastic solutions of sinusoidal contact for elastic and elastic-perfectly plastic materials Saha et al., 2016; Saha and Jackson, 2016; Wang et al., 2016], and few works account for strain hardening in sinusoidal or wavy surfaces. Liu and Proudhon [2014, 2016] recently established a force-deformation law for sinusoidal asperities (with shape ratio $\xi \in[0.01,0.20]$ ) before and after the onset of plastic deformation Liu and Proudhon, 2014], and they distinguished the contact evolution characteristics of a sinusoidal asperity with a shape ratio of 0.10 Liu and Proudhon, 2016]. In their work, the contact behavior of sinusoidal asperities with shape ratios greater than 0.40 was investigated as a complementary study for the simulation of rough surface contact; besides, for alloy surfaces, the five empirical equations of force-deformation were established according to the deformation evolution characteristics of sinusoidal contact [Liu and Proudhon, 2016], and the actual surfaces were simulated by a contact model with the consideration of the statistical height distribution of surface asperities.

In this paper, a rough contact model is proposed to study the interfacial behavior of $18 \mathrm{CrMo} 4$ steel surfaces. An elastic-plastic contact behavior of one sinusoidal pair is firstly built, and then the contact stiffness of rough surfaces is calculated using statistics, based on which, the effects of asperity parameters on interface stiffness are further discussed. The proposed model can be applied on the design of a required interface by manipulating roughness parameters.

\section{Statistical Model of Normal Contact Stiffness}

\section{1. $3 D$ sinusoidal asperity}

In microscopic observation, the roughness of an actual surface can be simplified into a composition of a large number of cones Hisakado, 1975], cylinders Li et al., 2018. spheres Greenwood and Williamson, 1966: Kogut and Etsion. 2002: Zhao et al., 2015; Lu et al., 2017, 2018], ellipsoids |Liu et al., 2015|, sinusoidal (or wavy) asperities Saha et al., 2016; Saha and Jackson, 2016; Wang et al., 2017; Liu, 2014; Liu and Proudhon, 2014, 2016; Westergaard, 1939; Gao et al., 2006; Johnson et al., 1985. and other shapes. For small load, the contact occurs at the tip of an asperity, and a spherical model can be used to simulate the contact behavior of asperities on a rough surface. For large load, some researchers Shah et al.., 2011: Jackson et al., 2008] suggested that sinusoidal contact can describe the surface asperity contact more accurately. Besides, Poon and Bhushan' surface measurements show 


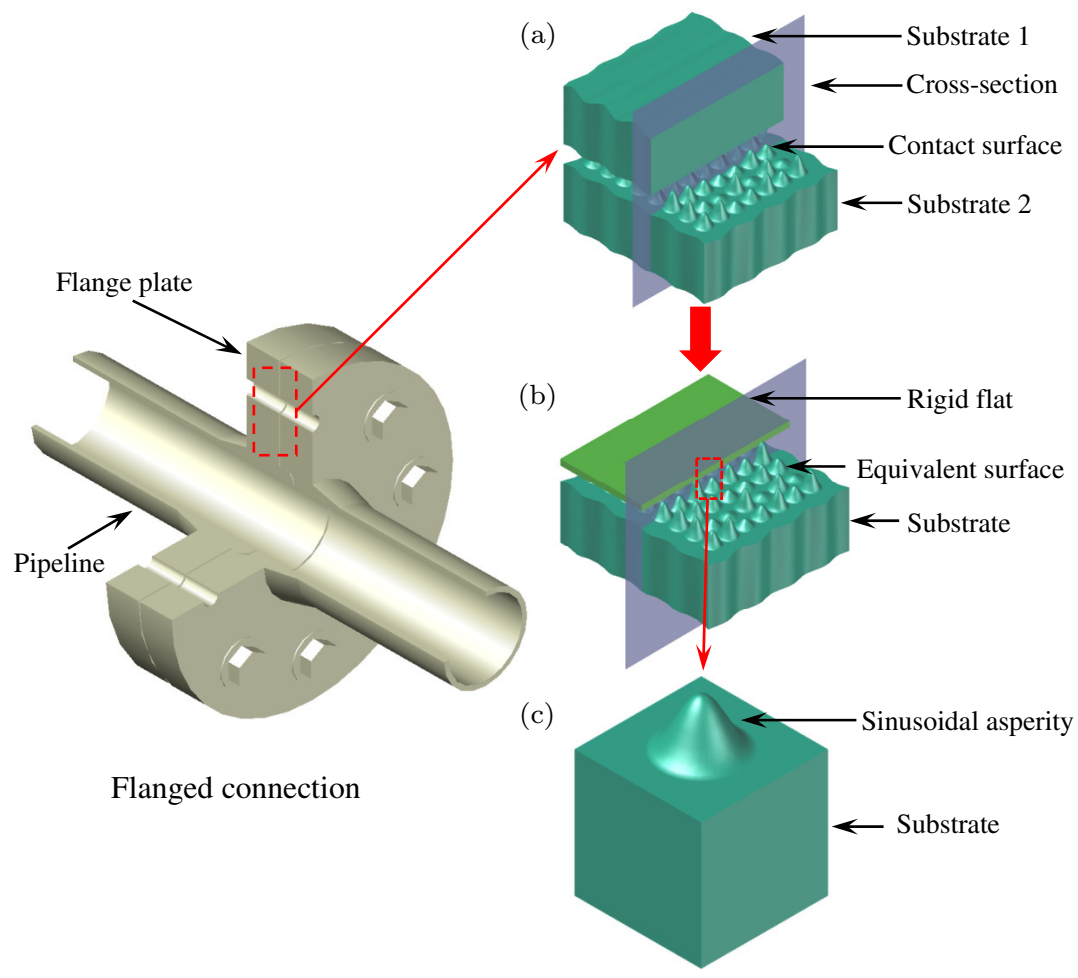

Fig. 1. Sketch of contact between two rough surfaces: (a) contact between two rough surfaces, (b) equivalent contact of two surfaces, and (c) 3D profile of sinusoidal asperity.

that the sinusoidal shape is close to the profile from surface measurement [Poon and Bhushan, 1995], and actual surfaces can be represented by either a Fourier series or a Weierstrass-Mandelbrot function Jackson and Streator, 2006; Wilson et al., 2010; Ciavarella et al., 2000], whose basic function is sinusoidal. Therefore, a sinusoidal (wavy) model is more practical than a spherical model.

Interfacial contact, such as flange connections shown in Fig. 1, is a common engineering problem. The contact behavior between flange plates is essentially the contact between the microstructures (i.e., asperities) on the substrates 1 and 2 (i.e., the flange plates 1 and 2) as in Fig. 1(a). Studies have demonstrated that the contact behavior between two rough surfaces is equivalent to that between an equivalent rough surface and a rigid flat as in Fig. 1(b) Greenwood and Tripp, 1970; Yu and Polycarpou, 2004]. Sinusoidal asperities are introduced to simulate the equivalent surface in this study. The 3D profile of a sinusoidal asperity is shown in Fig. 1(c).

The contact between a single sinusoidal asperity and a rigid flat is shown in Fig. 2. The profile curve of a sinusoidal asperity (2D) can be represented in Eq. (1):

$$
z(x)=\frac{h}{2}[\cos (2 \pi x / \lambda)+1] \text { for }(-\lambda / 2 \leq x \leq \lambda / 2),
$$




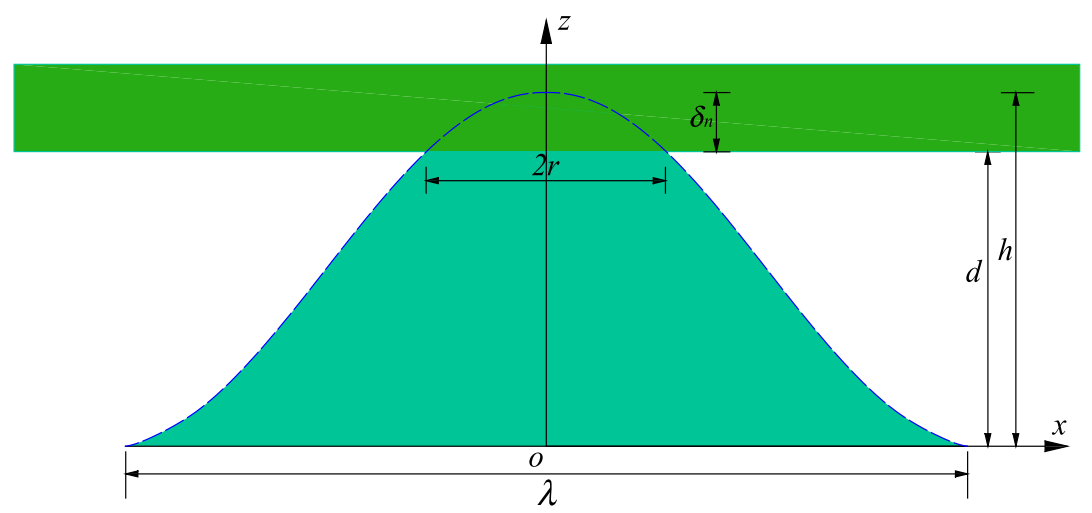

Fig. 2. Contact between a single asperity and a rigid smooth flat (oxz is a Cartesian coordinate system, $o$ is the coordinate origin at the top of the substrate): where $r$ denotes the radius of the contact; $d$ denotes the distance from the rigid flat to the top surface of the substrate; $\delta_{n}$ denotes the normal deformation of asperity, $\delta_{n}=h-d$.

where $h$ and $\lambda$ are the height and wavelength (or width) of a sinusoidal asperity, respectively.

\subsection{Power-law hardening property}

When 18CrMo4 steel undergoes plastic deformation, the plastic behavior obeys the $J_{2}$ flow theory and satisfies a power hardening law represented by the classical Ramberg-Osgood function [Ramberg and Osgood, 1943]. The relationship between the strain $\varepsilon$ and the stress $\sigma$ is as follows:

$$
\sigma=\left\{\begin{array}{lr}
E \varepsilon & \sigma \leq \sigma_{y} \\
\sigma_{y}\left[\left(E / \sigma_{y}\right) \varepsilon\right]^{n} & \text { when } \begin{array}{l}
\sigma>\sigma_{y}
\end{array},
\end{array},\right.
$$

where $E$ and $\sigma_{y}$ are the Young's modulus and yield strength of the material, respectively; and $n$ is the strain hardening exponent, ranging from 0 to 1 . The mechanical properties of 18CrMo4 steel are: Young's modulus $E=197.60 \mathrm{GPa}$, yield strength $\sigma_{u}=0.469 \mathrm{GPa}$, Poisson's ratio $\nu=0.29$ and strain hardening exponent $n=0.15$ Zhao et al., 2015].

\subsection{Finite element model}

The following three assumptions are made in this study for the efficiency of the simulation:

(a) Substrate deformation is not taken into account (see Appendix A).

(b) Interactions between adjacent asperities on the rough surface are ignored.

(c) Friction and adhesion between contact surfaces are not considered.

For most engineering surfaces, the roughness amplitude (asperity height $h$ ) is smaller than the width (wavelength $\lambda$ ), resulting from the surface spectrum of the 


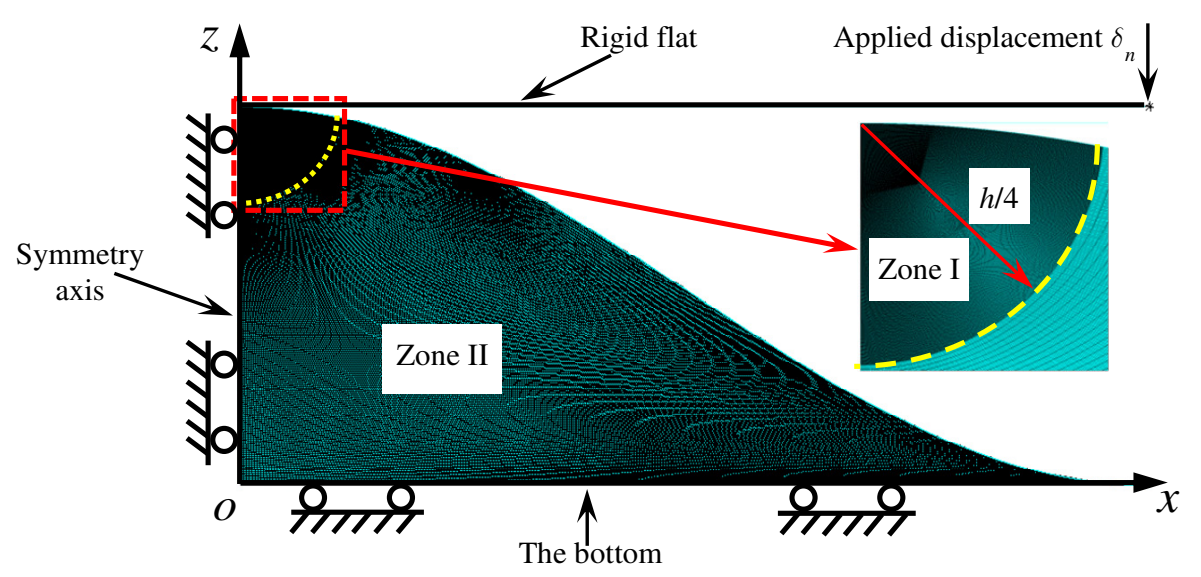

Fig. 3. FEM of sinusoidal asperity (Shape ratio $\xi=0.20, \lambda=1 \mathrm{~mm}$ ).

representative part. Generally, $\xi \in[0.02,0.20]$ Gao et al., 2006], and for a polished alloy $\xi \in[0.01,0.40]$ Yastrebov et al., 2011]. Since surfaces with larger ratios $\xi$ widely exist, asperities with different shape ratios are adopted to simulate the contact behavior of rough surfaces in this study, including $\xi=0.20,0.40,0.60$, and 1.00 .

As shown in Fig. 3. a line and a half-sinusoidal plane $(0 \leq x \leq \lambda / 2,0 \leq z \leq h)$ is used to represent a rigid flat and a deformable 3D sinusoidal asperity according to the axisymmetry of sinusoidal asperity. The origin $o, x$-axis, and $z$-axis of the Cartesian coordinate system are the center, bottom, and symmetry axis of the sinusoidal asperity, respectively.

For a small load, contact area between rough surfaces occurs firstly at the tip of an asperity. To ensure the numerical accuracy and further improve the computational efficiency, the half-sinusoidal plane is divided into two zones, as shown in Fig. 3. Zone I within a distance of $h / 4$ from the sinusoidal tip is the focal monitoring area with mesh size of $\lambda \times 10^{-4}$. Zone II outside the distance of $h / 4$ is coarsely meshed with the element size of $3 \lambda \times 10^{-3}$. Detail of the mesh sensitivity analysis is presented in Appendix B. Then, the half-sinusoidal plane is discretized by eight-node PLANE183 axisymmetric elements on the finite element software platform ANSYS R16.0. The contact is simulated with 2D surface-tosurface contact elements (CONTA172) and a single nonflexible two-node target element (TARGE169). Finally, the resulting mesh consists of 179403 PLANE183 elements and 503 CONTA172 elements. Compared with the normal Lagrange method and multipoint constraint method, the augmented Lagrange method is more suitable for contact problems without friction or adhesion and has a more efficient convergence rate for the contact calculation, which is thus used to calculate the contact in this study. In the numerical simulation, the force-deformation response of the sinusoidal asperity model needs to be consistent with that of an actual loaded asperity, 
and the boundary conditions of the asperity model Kogut and Etsion, 2002: Zhao et al., 2015] in this study are:

(a) The nodes at the bottom of the sinusoidal asperity are fixed in the $z$-direction, i.e., $U_{z}(x, 0)=0$.

(b) An axisymmetric boundary condition is applied at the axis of symmetry, i.e., $U_{x}(0, z)=0$.

(c) Using the contact wizard, a pilot node is generated at the right end of the rigid flat to control the degrees of freedom of the rigid flat.

(d) The vertical displacement $\delta_{n}$ is applied to the pilot node.

\subsection{Numerical results}

\subsubsection{Dimensionless results}

To make the empirical equation simple and available, the simulated normal deformation $\delta_{n}$ and simulated normal force $f_{n}$ are normalized by the critical deformation $\delta_{n, c}$ and critical force $f_{n, c}$, respectively, when the sphere just yields, i.e., $\delta_{n}^{*}=\delta_{n} / \delta_{n, c}$ and $f_{n}^{*}=f_{n} / f_{n, c}$. According to the Hertz theory Hertz, 1882], the critical deformation can be calculated by Eq. (3):

$$
\delta_{n, c}=\left(\frac{3 \pi p_{m}}{4 E^{*}}\right)^{2} R_{s} .
$$

The critical force is shown in Eq. (4):

$$
f_{n, c}=\frac{4 E^{*}}{3}\left(R_{s} \delta_{n, c}^{3}\right)^{1 / 2}=\left(\frac{3 R_{s}}{4 E^{*}}\right)^{2}\left(\pi p_{m}\right)^{3},
$$

where $p_{m}$ is the average contact pressure; $R_{s}$ is the curvature radius of the asperity summit; $H$ is the hardness of the softer material in contact; and $E^{*}$ is the effective modulus of elasticity: $1 / E^{*}=\left(1-v_{1}^{2}\right) / E_{1}+\left(1-v_{2}^{2}\right) / E_{2}$, where $E_{1}, E_{2}, \nu_{1}$, and $\nu_{2}$ are the Young's moduli and Poisson's ratios of material 1 and material 2, respectively.

For the contact between a surface of a sinusoidal asperity and a rigid flat with $E_{2} \rightarrow \infty$, the curvature radius of the sinusoidal asperity summit is $R_{s}=\lambda^{2} /\left(2 \pi^{2} h\right)$. When the asperity just yields, the maximum Hertz contact pressure can be as high as $p_{\max }=0.60 \mathrm{H}$, i.e., $p_{m}=2 p_{\max } / 3=0.40 \mathrm{H}$ Tabor, 1951 . The hardness of a metal material $H=2.80 \sigma_{y}$ Kogut and Etsion, 2002; Tabor, 1951; Jamari and Schipper, 2006], and the hardness of $18 \mathrm{CrMo} 4$ steel is $1313.2 \mathrm{MPa}$.

For real surfaces, the areal contact deformation is determined by the parameters of the surface morphology and the mechanical properties of the material, which can be represented by the plasticity index in Eq. (5) [Greenwood and Williamson, 1966]:

$$
\psi=\frac{E^{*}}{H}\left(\frac{\sigma_{z}}{R_{s, m}}\right)^{1 / 2},
$$

where $\sigma_{z}$ is the standard deviation of the asperity heights and $R_{s, m}$ is the mean curvature radius of the asperity summits on the rough surface. 


\subsubsection{Deformation}

a. Analysis of deformation The von Mises yield criterion is used during the contact. Liu and Proudhon [2016] characterized the deformation by the plastic zone inside the asperity, as shown in Fig. 4. The five deformation phases of a sinusoidal asperity are illustrated in Fig. 5. It can be seen from Figs. 4 and 5 that the four transitional boundaries between the deformation phases are:

(i) At the moment when plastic deformation occurs inside the asperity, the plastic region is surrounded by elastic regions, corresponding to the first elastic-plastic critical point $T_{1}$, for which the value $\delta_{n, 1}^{*}$ is listed in Table 1. The equivalent plastic strain field [Kogut and Etsion, 2002; Liu and Proudhon, 2016] at this instant is shown in Fig. 4 (a).

(ii) The moment that the plastic zone reaches the contact surface for the first time marks the elastic limit of the contact surface, which is indicated by the second elastic-plastic critical point $T_{2}$, for which the value $\delta_{n, 2}^{*}$ is listed in Table 1 The equivalent plastic strain field is shown in Fig. 4(b).

(iii) The moment that the whole contact surface experiences plastic deformation for the first time is indicated by the first plastic critical point $T_{3}$, for which the value $\delta_{n, 3}^{*}$ is listed in Table 1. The equivalent plastic strain field is shown in Fig. 4(c).

(iv) The moment that the mean contact pressure reaches the hardness of the material is indicated by the fully plastic critical point $T_{4}$, for which the value $\delta_{n, 4}^{*}$ is listed in Table 1, The equivalent plastic strain field is shown in Fig. 4(d).

b. Empirical equations According to the four transitional boundaries above, the deformation of a sinusoidal asperity is divided into five deformation phases: the elastic, the first elastic-plastic, the second elastic-plastic, the first plastic and fully plastic deformation. The deformation ranges for each phase are $\delta_{n}^{*} \leq \delta_{n, 1}^{*}, \delta_{n, 1}^{*}<$ $\delta_{n}^{*} \leq \delta_{n, 2}^{*}, \delta_{n, 2}^{*}<\delta_{n}^{*} \leq \delta_{n, 3}^{*}, \delta_{n, 3}^{*}<\delta_{n}^{*} \leq \delta_{n, 4}^{*}$ and $\delta_{n}^{*}>\delta_{n, 4}^{*}$.

Before the sinusoidal asperity undergoes plasticity, the force-deformation response follows a power law relation, as reported in previous studies [Liu and Proudhon, 2014]. Namely, under the condition of the deformation $\delta_{n}^{*} \leq \delta_{n, 3}^{*}$, the empirical equation can be expressed by Eq. (6):

$$
f_{n}^{*}=e_{i} \delta_{n}^{* c_{i}}
$$

where $e_{i}$ and $c_{i}$ are the coefficient and exponent of a power law function, respectively. The values of $e_{i}$ and $c_{i}$ are listed in Tables 2 and 3 , respectively.

After the strain on the entire contact region exceeds the yield strain, the asperity completely goes into the plastic phase, i.e., the entire contact zone is plastic. However, the mean contact pressure on the contact surface has not reach the hardness value of the metal material. As $\delta_{n}^{*}$ increases, the plastic zone continues to expand. When the mean contact pressure reaches the hardness, the sinusoidal asperity enters the fully plastic phase. The force-deformation response follows a linear relation, 


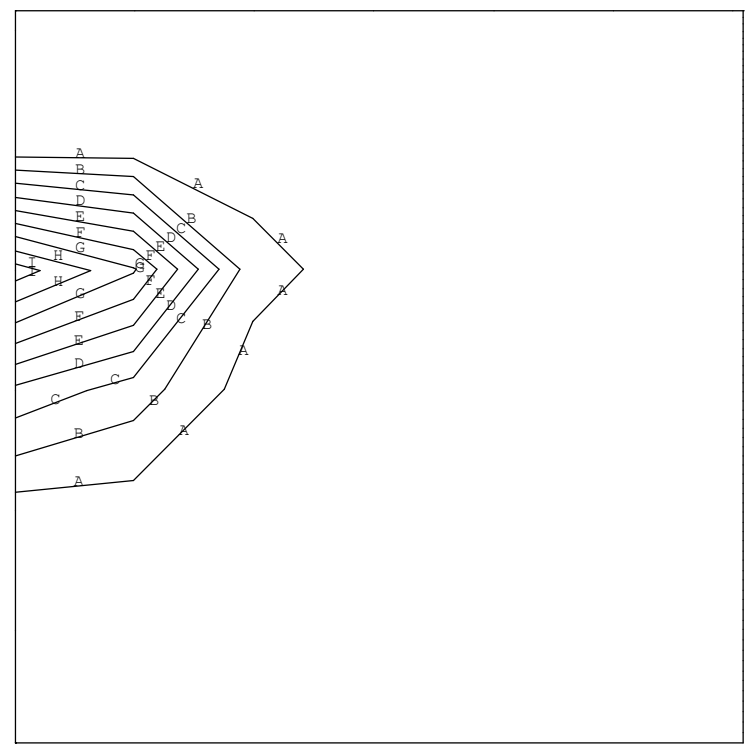

$$
\begin{aligned}
& \text { ANYS Release } 16.0 \\
& =.184 \mathrm{E}-05 \\
& =.551 \mathrm{E}-05 \\
& =.919 \mathrm{E}-05 \\
& =.129 \mathrm{E}-04 \\
& =.165 \mathrm{E}-04 \\
& =.202 \mathrm{E}-04 \\
& =.239 \mathrm{E}-04 \\
& =.276 \mathrm{E}-04 \\
& =.312 \mathrm{E}-04
\end{aligned}
$$

(a)

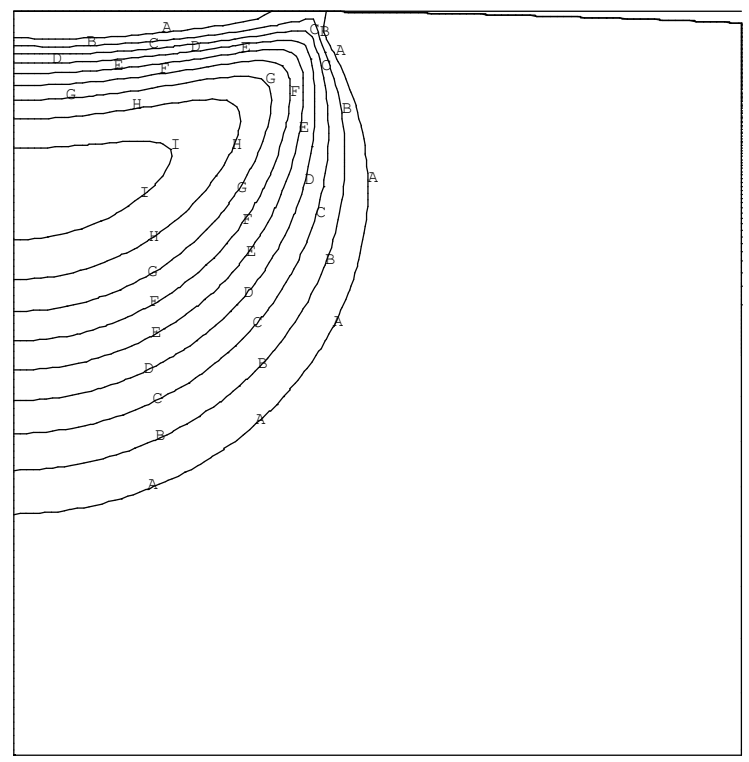

$$
\begin{array}{ll}
\text { ANSYS Release } & 16.0 \\
\mathrm{~A} \quad=.310 \mathrm{E}-03 \\
\mathrm{~B} \quad=.929 \mathrm{E}-03 \\
\mathrm{C} \quad=.001549 \\
\mathrm{D} \quad=.002168 \\
\mathrm{E} \quad=.002787 \\
\mathrm{~F} \quad=.003407 \\
\mathrm{G} \quad=.004026 \\
\mathrm{H} \quad=.004646 \\
\mathrm{I} \quad=.005265
\end{array}
$$

(b)

Fig. 4. Schematic of evolution of equivalent plastic strain field when $\lambda=1 \mathrm{~mm}$ and $\xi=0.20$ : (a) The moment that the plastic zone appears in the sinusoidal asperity for the first time, $T_{1}$ $\left(\delta_{n, 1} / h=3.76 \times 10^{-5}\right)$, (b) The moment that plastic zone just reaches the contact surface, $T_{2}\left(\delta_{n, 2} / h=3.29 \times 10^{-4}\right)$, (c) The moment that the whole contact surface experiences plastic deformation, $T_{3}\left(\delta_{n, 3} / h=6.32 \times 10^{-3}\right)$, and (d) The moment that the mean contact pressure reaches the hardness, i.e., full plastic deformation occurs, $T_{4}\left(\delta_{n, 4} / h=1.81 \times 10^{-2}\right)$. 


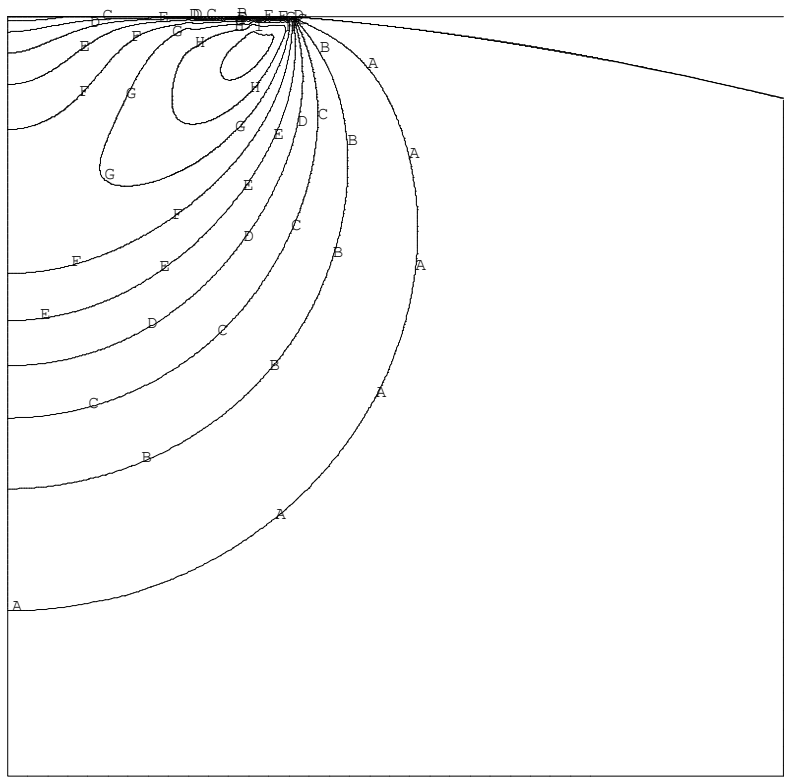

ANSYS Release 16.0

$\mathrm{A}=.002588$

$=.002588$

$=.012938$

$=.018114$

$=.023289$

$=.028465$

$=.03364$

$=.038815$

(c)

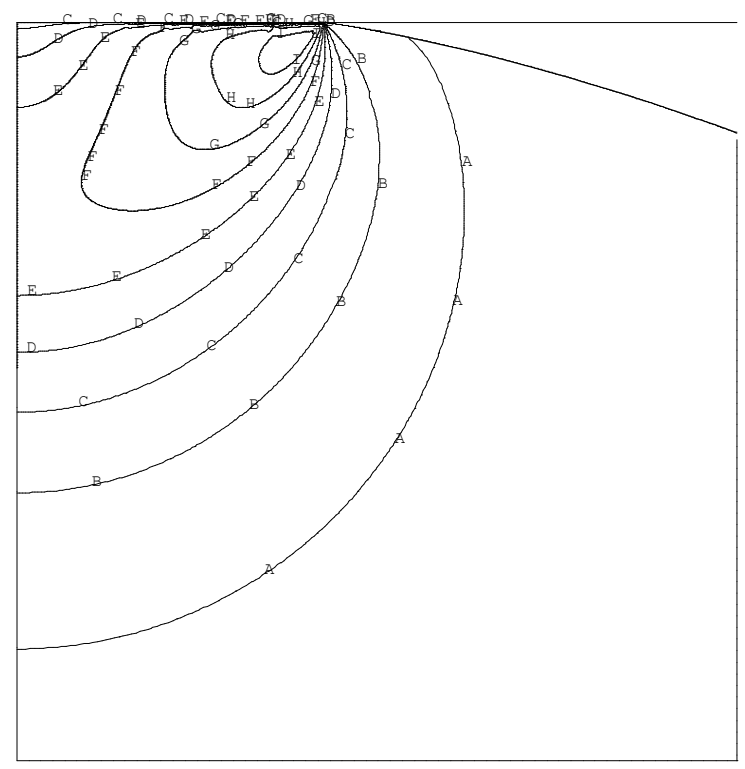

ANSYS Release 16.0

$=.005446$

$=.016337$

$=.027229$

$=.03812$

$=.049012$

$=.059903$

$=.070795$

$=.081686$

$=.092578$

(d)

Fig. 4. (Continued) 


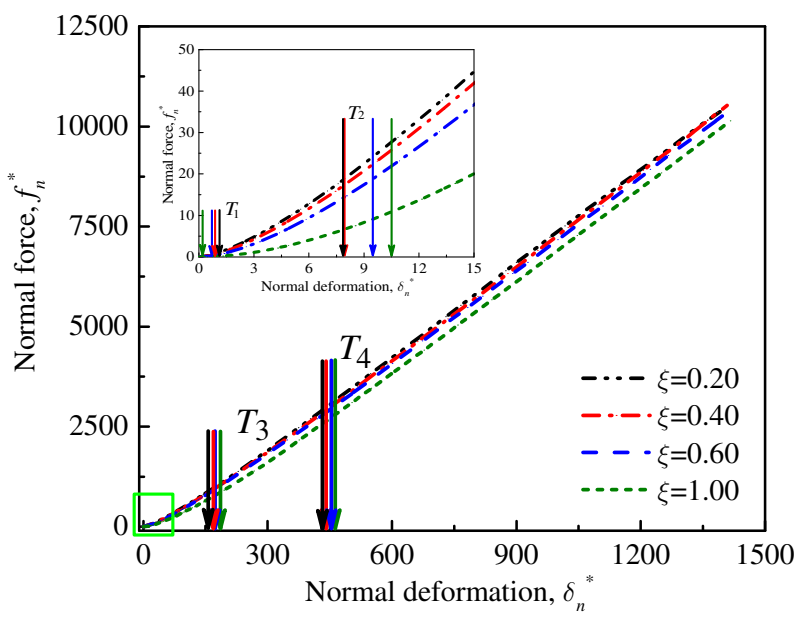

Fig. 5. (Color online) Variation of normalized normal force $f_{n}^{*}$ with normal deformation $\delta_{n}^{*}$ (the solid vertical arrows indicates transitional moments, and the content in the green frame is amplified in the upper left panel).

Table 1. The value of the critical deformation $\delta_{n, i}^{*}$.

\begin{tabular}{cccc}
\hline The critical deformation, $\delta_{n, i}^{*}$ & Slope, $a_{i}$ & Intercept, $b_{i}$ & $R^{2}$ \\
\hline$\delta_{n, 1}^{*}$ & -0.98 & 1.10 & 0.99 \\
$\delta_{n, 2}^{*}$ & 3.63 & 6.77 & 0.92 \\
$\delta_{n, 3}^{*}$ & 27.23 & 148.57 & 0.92 \\
$\delta_{n, 4}^{*}$ & 42.83 & 425.36 & 0.99 \\
\hline
\end{tabular}

Note: $\delta_{n}^{*}, i=a_{i} \xi+b_{i}, i=1,2,3$, and $4 . R^{2}$ is the coefficient of determination, which is used to evaluate the fitting degree, and $R^{2}>0.90$ is desirable.

Table 2. The value of the coefficient $e_{i}$.

\begin{tabular}{lcccc}
\hline$e_{i}$ & $M_{i}$ & $N_{i}$ & $G_{i}$ & $R^{2}$ \\
\hline$e_{1}$ & 0 & -0.86 & 1.10 & 0.97 \\
$e_{2}$ & 0 & -1.09 & 1.25 & 0.99 \\
$e_{3}$ & -1.19 & 0.04 & 1.75 & 0.99 \\
$e_{4}$ & 0 & -0.08 & 7.21 & 0.92 \\
$e_{5}$ & 0 & -0.12 & 7.80 & 0.96 \\
\hline
\end{tabular}

Note: $e_{i}=M_{i} \xi^{2}+N_{i} \xi+G_{i}, i=1,2,3,4$, and 5. $R^{2}$ is the coefficient of determination, which is used to evaluate the fitting degree, and $R^{2}>0.90$ is desirable.

as shown in Fig. 5, as reported in previous studies Liu and Proudhon, 2014]. Namely, under the condition of the deformation $\delta_{n}^{*}>\delta_{n, 3}^{*}$, the empirical equation can be expressed by Eq. (7):

$$
f_{n}^{*}=e_{i} \delta_{n}^{*}+c_{i}
$$

where $e_{i}$ and $c_{i}$ are the coefficient and intercept of a linear function, respectively. The values of $e_{i}$ and $c_{i}$ are listed in Tables 2 and 3 , respectively. 
Table 3. The value of the coefficient $c_{i}$.

\begin{tabular}{llrrr}
\hline$c_{i}$ & $P_{i}$ & \multicolumn{1}{c}{$Q_{i}$} & $O_{i}$ & $R^{2}$ \\
\hline$c_{1}$ & 0.69 & 0.58 & 1.39 & 0.99 \\
$c_{2}$ & 0.38 & 0.03 & 1.39 & 0.99 \\
$c_{3}$ & 0.26 & -0.11 & 1.25 & 0.99 \\
$c_{4}$ & 0 & -341.63 & -156.39 & 0.92 \\
$c_{5}$ & 0 & -402.15 & -377.28 & 0.95 \\
\hline
\end{tabular}

Note: $c_{i}=P_{i} \xi^{2}+Q_{i} \xi+O_{i}, i=1,2,3,4$, and $5 . R^{2}$ is the coefficient of determination, which is used to evaluate the fitting degree, and $R^{2}>0.90$ is desirable.

\subsubsection{Elastic-plastic contact law of a single asperity}

According to the Hooke's law, normal contact stiffness is the derivative of normal contact force, which is a function of $\delta_{n}$, as shown in Eq. (8):

$$
k_{n}\left(\delta_{n}\right)=\frac{d f_{n}\left(\delta_{n}\right)}{d \delta_{n}}
$$

Based on the simulation results of the contact between a single sinusoidal asperity with a height of $z$ and a rigid smooth flat, an empirical equation of the normal contact force $f_{n}$ can be derived as in Eq. (99):

$$
f_{n}= \begin{cases}f_{n, c} \times\left[e_{1} \times\left(\frac{\delta_{n}}{\delta_{n, c}}\right)^{c_{1}}\right] & 0<\delta_{n} \leq \delta_{n, 1} \\ f_{n, c} \times\left[e_{2} \times\left(\frac{\delta_{n}}{\delta_{n, c}}\right)^{c_{2}}\right] & \delta_{n, 1}<\delta_{n} \leq \delta_{n, 2} \\ f_{n, c} \times\left[e_{3} \times\left(\frac{\delta_{n}}{\delta_{n, c}}\right)^{c_{3}}\right] & \text { when } \delta_{n, 2}<\delta_{n} \leq \delta_{n, 3} \\ f_{n, c} \times\left[e_{4} \times\left(\frac{\delta_{n}}{\delta_{n, c}}\right)+c_{4}\right] & \delta_{n, 3}<\delta_{n} \leq \delta_{n, 4} \\ f_{n, c} \times\left[e_{5} \times\left(\frac{\delta_{n}}{\delta_{n, c}}\right)+c_{5}\right] & \delta_{n}>\delta_{n, 4} .\end{cases}
$$

Therefore, the normal contact stiffness $k_{n}$ of a sinusoidal asperity can be expressed as in Eq. (10):

$$
k_{n}= \begin{cases}f_{n, c} \times\left[\frac{e_{1} \times c_{1}}{\delta_{n, c}} \times\left(\frac{\delta_{n}}{\delta_{n, c}}\right)^{c_{1}-1}\right] & 0 \leq \delta_{n} \leq \delta_{n, 1} \\ f_{n, c} \times\left[\frac{e_{2} \times c_{2}}{\delta_{n, c}} \times\left(\frac{\delta_{n}}{\delta_{n, c}}\right)^{c_{2}-1}\right] & \delta_{n, 1}<\delta_{n} \leq \delta_{n, 2} \\ f_{n, c} \times\left[\frac{e_{3} \times c_{3}}{\delta_{n, c}} \times\left(\frac{\delta_{n}}{\delta_{n, c}}\right)^{c_{3}-1}\right] & \delta_{n, 2}<\delta_{n} \leq \delta_{n, 3} \\ f_{n, c} \times \frac{e_{4}}{\delta_{n, c}} & \delta_{n, 3}<\delta_{n} \leq \delta_{n, 4} \\ f_{n, c} \times \frac{e_{5}}{\delta_{n, c}} & \delta_{n}>\delta_{n, 4},\end{cases}
$$


where $\delta_{n}=z-d$, wherein $d$ denotes the separation distance between two contact surfaces; $\delta_{n, 1}, \delta_{n, 2}, \delta_{n, 3}$ and $\delta_{n, 4}$ are the first elastic-plastic, the second elasticplastic, the first plastic and the fully plastic critical deformation of the sinusoidal asperity, respectively.

\subsection{Statistical contact model of a rough surface}

Morphology measurements show that asperity heights follow a Gaussian distribution for many surfaces Greenwood and Williamson, 1966]. According to the probability density function $\varphi(z)$, the total number of asperities participating contact at height $z$ on a rough surface can be calculated by Eq. (11):

$$
N_{\mathrm{con}}=\eta A_{\mathrm{sur}} \int_{d}^{\infty} \varphi(z) d z=\eta A_{\mathrm{sur}} \int_{d}^{\infty} \frac{1}{\sigma_{z} \sqrt{(2 \pi)}} \exp \left[-\frac{1}{2}\left(\frac{z-\mu_{z}}{\sigma_{z}}\right)^{2}\right] d z
$$

where $A_{\text {sur }}$ is the area of a whole rough surface; $\eta$ is the surface density of asperities; $\mu_{z}$ and $\sigma_{z}$ are the mean value of the asperity heights and the standard deviation of the asperity heights, respectively; $\mu_{z}$ and $\sigma_{z}$ are replaced with the average roughness $R_{a}$ and the root mean square roughness (i.e., RMS) $R_{q}$ in Secs. 3.2, 3.3, and 3.4, respectively.

The normal pressure $P_{n}$ and normal contact stiffness $K_{n}$ of the whole contact surface are determined by combining the elastic-plastic contact response of a single asperity in Eqs. (9) and (10), as well as the total number of asperities participating contact in Eq. (11). The normal contact force and normal contact stiffness of the whole surface are divided by the area $A_{\text {sur }}$, and the normal pressure $P_{n}$ of a rough surface is expressed in Eq. (12):

$$
\begin{aligned}
P_{n}(d)= & \eta\left\{\int_{d}^{d+\delta_{n, 1}} f_{n, e} \varphi(z) d z+\int_{d+\delta_{n, 1}}^{d+\delta_{n, 2}} f_{n, e p, 1} \varphi(z) d z+\int_{d+\delta_{n, 2}}^{d+\delta_{n, 3}} f_{n, e p, 2} \varphi(z) d z\right. \\
& \left.+\int_{d+\delta_{n, 3}}^{d+\delta_{n, 4}} f_{n, p, 1} \varphi(z) d z+\int_{d+\delta_{n, 4}}^{+\infty} f_{n, f p} \varphi(z) d z\right\} \\
= & \eta\left\{\int_{d}^{d+\delta_{n, 1}} f_{n, c} \times e_{1}\left(\frac{z-d}{\delta_{n, c}}\right)^{c_{1}} \varphi(z) d z\right. \\
& +\int_{d+\delta_{n, 1}}^{d+\delta_{n, 2}} f_{n, c} \times e_{2}\left(\frac{z-d}{\delta_{n, c}}\right)^{c_{2}} \varphi(z) d z \\
& +\int_{d+\delta_{n, 2}}^{d+\delta_{n, 3}} f_{n, c} \times e_{3}\left(\frac{z-d}{\delta_{n, c}}\right)^{c_{3}} \varphi(z) d z
\end{aligned}
$$




$$
\begin{aligned}
& +\int_{d+\delta_{n, 3}}^{d+\delta_{n, 4}} f_{n, c} \times\left[e_{4}\left(\frac{z-d}{\delta_{n, c}}\right)+c_{4}\right] \varphi(z) d z \\
& \left.+\int_{d+\delta_{n, 4}}^{+\infty} f_{n, c} \times\left[e_{5}\left(\frac{z-d}{\delta_{n, c}}\right)+c_{5}\right] \varphi(z) d z\right\} .
\end{aligned}
$$

Furthermore, the normal contact stiffness $K_{n}$ of a rough surface is expressed in Eq. (13):

$$
\begin{aligned}
K_{n}(d)= & \left\{\int_{d}^{d+\delta_{n, 1}} k_{n, e} \varphi(z) d z+\int_{d+\delta_{n, 1}}^{d+\delta_{n, 2}} k_{n, e p, 1} \varphi(z) d z+\int_{d+\delta_{n, 2}}^{d+\delta_{n, 3}} k_{n, e p, 2} \varphi(z) d z\right. \\
& \left.+\int_{d+\delta_{n, 3}}^{d+\delta_{n, 4}} k_{n, p, 1} \varphi(z) d z+\int_{d+\delta_{n, 4}}^{+\infty} k_{n, f p} \varphi(z) d z\right\} \\
= & \eta \int_{d}^{d+\delta_{n, 1}}\left[f_{n, c} \times \frac{e_{1} \times c_{1}}{\delta_{n, c}} \times\left(\frac{z-d}{\delta_{n, c}}\right)^{c_{1}-1}\right] \varphi(z) d z \\
& +\int_{d+\delta_{n, 1}}^{d+\delta_{n, 2}}\left[f_{n, c} \times \frac{e_{2} \times c_{2}}{\delta_{n, c}} \times\left(\frac{z-d}{\delta_{n, c}}\right)^{c_{2}-1}\right] \varphi(z) d z \\
& +\int_{d+\delta_{n, 2}}^{d+\delta_{n, 3}}\left[f_{n, c} \times \frac{e_{3} \times c_{3}}{\delta_{n, c}} \times\left(\frac{z-d}{\delta_{n, c}}\right)^{c_{3}-1}\right] \varphi(z) d z \\
& +\int_{d+\delta_{n, 3}}^{d+\delta_{n, 4}}\left[f_{n, c} \times \frac{e_{4}}{\delta_{n, c}}\right] \varphi(z) d z \\
& \left.+\int_{d+\delta_{n, 4}}^{+\infty}\left[f_{n, c} \times \frac{e_{5}}{\delta_{n, c}}\right] \varphi(z) d z\right\} \cdot(13)
\end{aligned}
$$

\subsection{Calculation of normal pressure and normal contact stiffness}

The procedure of calculating the normal pressure $P_{n}$ and normal contact stiffness $K_{n}$ is shown in Fig. 6. First, morphology parameters $\mu_{z}$ (or $\left.R_{a}\right), \sigma_{z}\left(\right.$ or $\left.R_{q}\right), \eta$ and $R_{s, m}$ are selected for the rough surface, with which the asperity heights follow a Gaussian distribution. Second, as $d$ decreases, the normal deformation of the contact surfaces is determined by $\delta_{n}=h_{\max }-d$ when $(z-d)$ becomes larger than 0 for the first time. The normal contact force and normal contact stiffness of the asperities with a height of $z$ on the whole contact surface are determined by combining the contact law of a single asperity (Eqs. (9) and (10) ) and the total number of asperities participating contact in the interval $z$ to $z-\Delta z$ (Eq. (11)). A step-size of $\Delta z=0.001$ $\left(h_{\max }-d_{\text {cer }}\right)$ for the probability calculation can satisfy the computational accuracy 


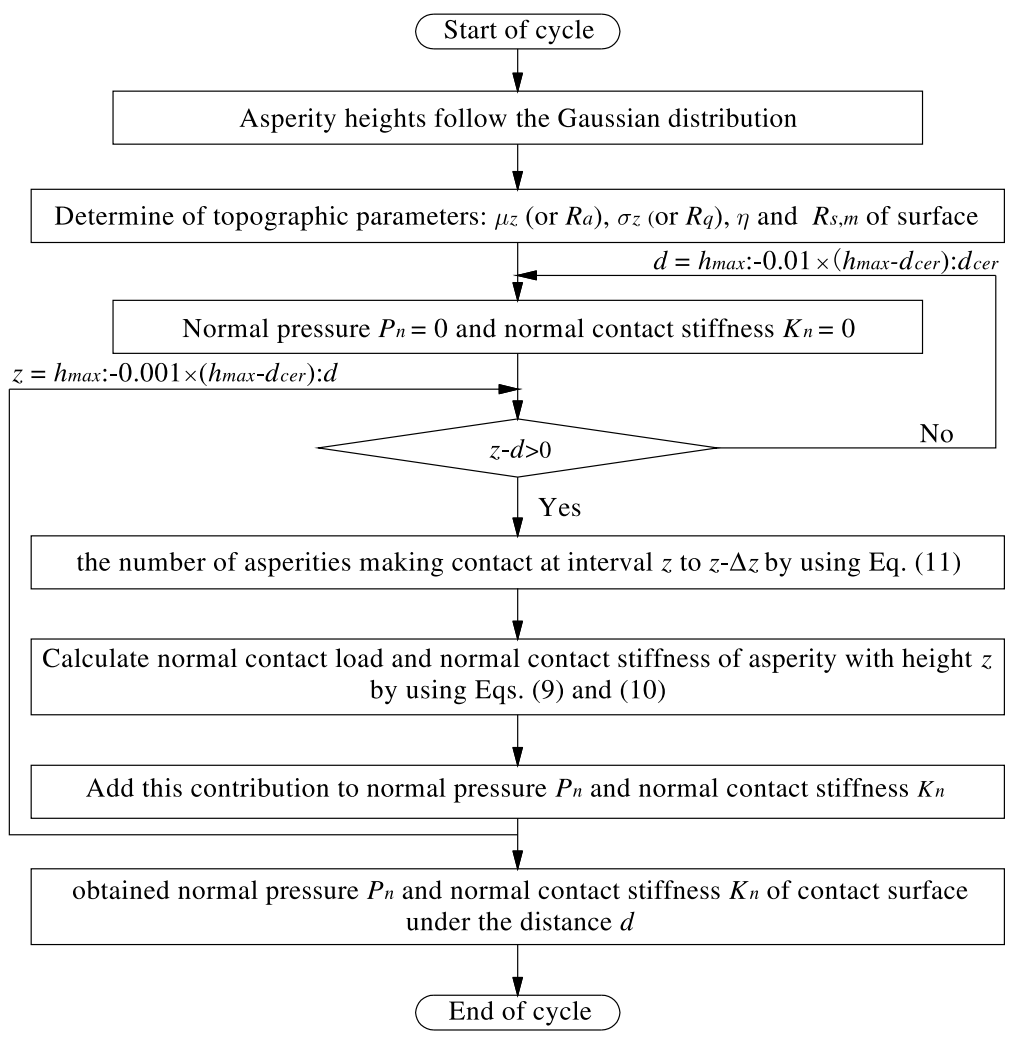

Fig. 6. Procedure to determine the normal pressure $P_{n}$ and normal contact stiffness $K_{n}$ of the contact surfaces.

of the normal pressure $P_{n}$ and normal contact stiffness $K_{n}$ of a rough surface. Then, such calculation iterates from $z=h_{\max }$ to $d$. Finally, when the distance is $d$, the normal pressure $P_{n}$ and normal contact stiffness $K_{n}$ of the whole contact surfaces are determined by summing up Eqs. (12) and (13). The calculation of the normal pressure $P_{n}$ and normal contact stiffness $K_{n}$ iterates from $d=h_{\max }$ to $d=d_{\text {cer }}$, where $d_{\text {cer }}$ is the terminal value of the calculation. The calculation is realized using Matlab script, which is provided with the electronic version on line.

\section{Results and Discussion}

\subsection{Contact behavior of rough surfaces with different shape ratios $\xi$}

The asperity shape ratio $\xi$ has great influence on the normal pressure $P_{n}$ and the normal contact stiffness $K_{n}$ of a rough surface. Four surfaces with shape ratios of $0.20,0.40,0.60$, and 1.00 are selected, the mean height of which is $0.31 \mu \mathrm{m}$ with the 
standard deviation of $6.20 \mu \mathrm{m}$, and the surface density of asperities is $40,194 / \mathrm{mm}^{2}$ Zhao et al., 2015].

Figure 7(a) shows the relationships between the normal deformation $\delta_{n}$ and the normal pressure $P_{n}$ for rough surfaces with different shape ratios $\xi$. The normal pressure nonlinearly increases with the increase of the normal deformation. For the same $\delta_{n}$, the normal pressure decreases as the shape ratio $\xi$ increases. Figure $7(\mathrm{~b})$ shows the relationships between the normal pressure $P_{n}$ and the normal contact stiffness $K_{n}$ for rough surfaces with different shape ratios $\xi$. The normal contact

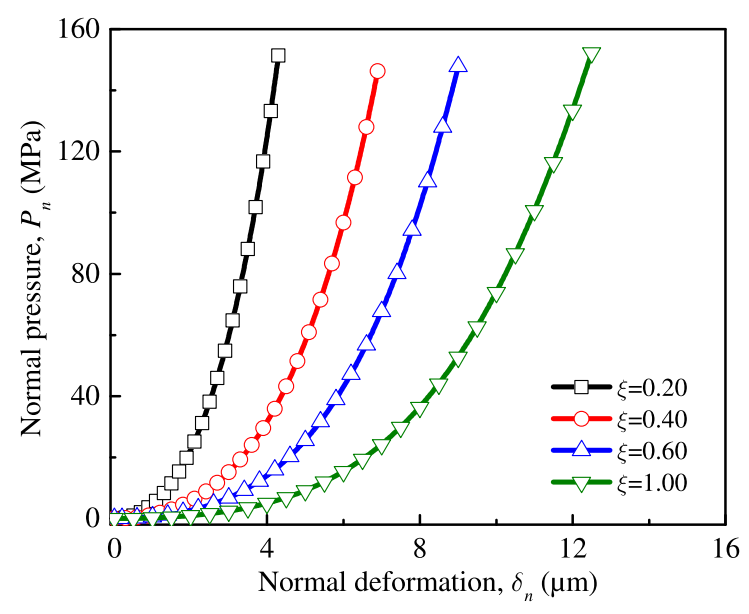

(a)

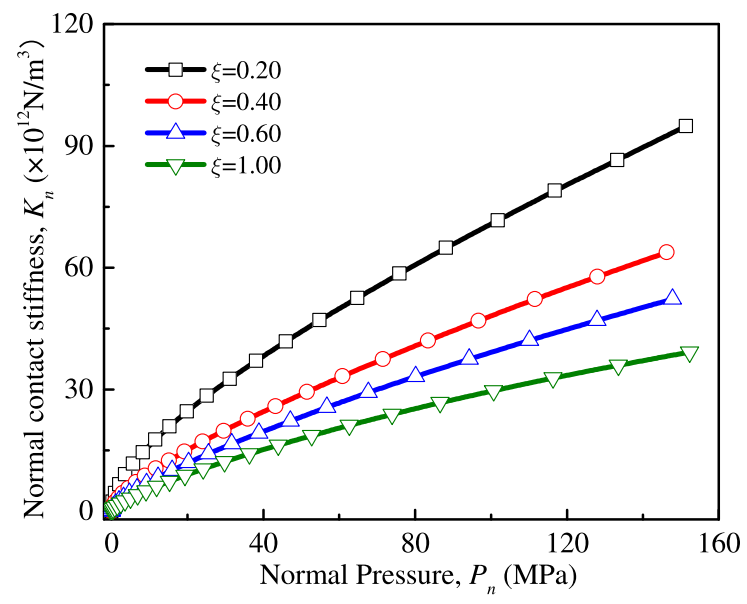

(b)

Fig. 7. Contact behavior of rough surfaces with different shape ratios $\xi$ : (a) the relationships between the normal deformation $\delta_{n}$ and the normal pressure $P_{n}$ and (b) the relationships between the normal pressure $P_{n}$ and the normal contact stiffness $K_{n}$. 
stiffness nonlinearly increases with the increase of the normal pressure. For the same $P_{n}$, the normal contact stiffness decreases as $\xi$ increases.

\subsection{Contact behavior of rough surfaces with different wavelengths $\lambda$}

The asperity wavelength $\lambda$ affects the normal pressure $P_{n}$ and the normal contact stiffness $K_{n}$ of a rough surface. The wavelength range of asperities on the rough surfaces is related to the surface treatment methods. In this study, the polished alloy from the study of Timsit [2006] with the wavelength ranging from $50 \mathrm{~nm}$ to $250 \mathrm{~nm}$ is analyzed. When the asperity width $\lambda=250 \mathrm{~nm}$, the surface density of asperities is at least $\eta=1.6 \times 10^{7} / \mathrm{mm}^{2}$. Five surfaces with wavelengths of 50,100 , 150,200 , and $250 \mathrm{~nm}$ are selected, for which the roughness are $R_{a}=0.01 \mu \mathrm{m}$ and $R_{q}=0.0125 \mu \mathrm{m}$ (i.e.. $R_{a} / R_{a}=1.25$ Thomas. 1999: Kumar and Rao. 2012: Vitanov et al., 2014]).

Figure 8 (a) shows the relationships of the normal deformation $\delta_{n}$ and the normal pressure $P_{n}$ for rough surfaces with different wavelengths $\lambda$. The normal pressure increases with the increase of the normal deformation. For the same $\delta_{n}$, the normal pressure increases as $\lambda$ increases. Figure 8(b) shows the relationships between the normal pressure and the normal contact stiffness for rough surfaces with different wavelengths $\lambda$. The normal contact stiffness increases as the normal pressure increases. For the same $P_{n}$, the normal contact stiffness increases as the wavelength $\lambda$ increases.

\subsection{Contact behavior of rough surfaces with different roughness $R_{a}$}

The roughness $R_{a}$ influences the normal pressure $P_{n}$ and the normal contact stiffness $K_{n}$ of a rough surface. The roughness grading requirements of mating surfaces in engineering are as follows:

(a) For general gears, $R_{a}=1.60 \mu \mathrm{m}$.

(b) For rough surfaces with centering and matching characteristics, $R_{a}=0.80 \mu \mathrm{m}$.

(c) For rough surfaces with long-term stability, $R_{a}=0.40 \mu \mathrm{m}$.

(d) For high-quality surfaces, $R_{a}=0.05 \mu \mathrm{m}$.

For rough surfaces with asperity heights following Gaussian distribution, statistics shows that the ratio of $R_{\alpha}$ to $R_{a}$ should be 1.25 Thomas, 1999: Kumar and Rao, 2012; Vitanov et al., 2014]. Hence, four surfaces with $R_{a}=1.60 \mu \mathrm{m}$ and $R_{q}=2.00 \mu \mathrm{m}, R_{a}=0.80 \mu \mathrm{m}$ and $R_{q}=1.00 \mu \mathrm{m}, R_{a}=0.40 \mu \mathrm{m}$ and $R_{q}=0.50 \mu \mathrm{m}$ and $R_{a}=0.05 \mu \mathrm{m}$, and $R_{q}=0.0625 \mu \mathrm{m}$ are selected, for which the mean curvature radius of asperity summits $R_{s, m}$ and the surface density of asperities $\eta$ are $21.08 \mu \mathrm{m}$ and $40,194 / \mathrm{mm}^{2}$ [Zhao et al., 2015], respectively.

Figure 9(a) shows the relationships between the normal deformation $\delta_{n}$ and the normal pressure $K_{n}$ for rough surfaces with different roughness $R_{a}$. For the 


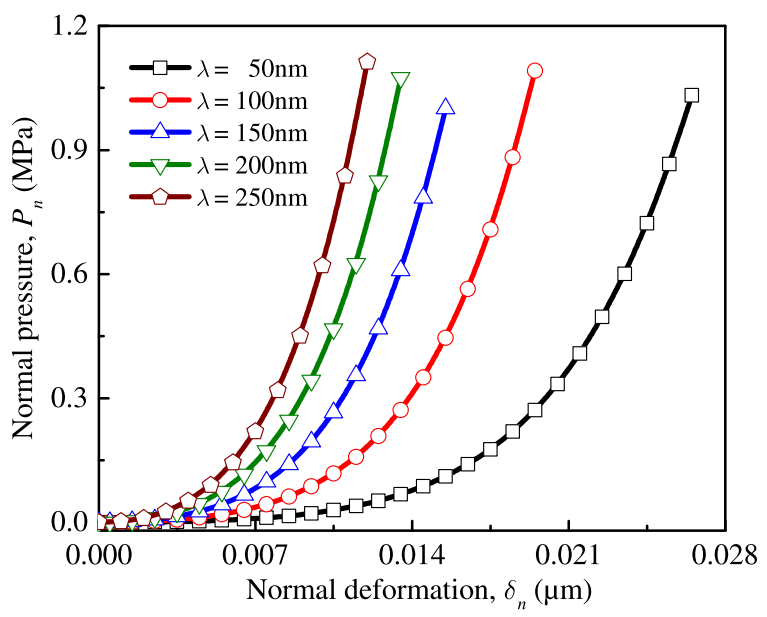

(a)

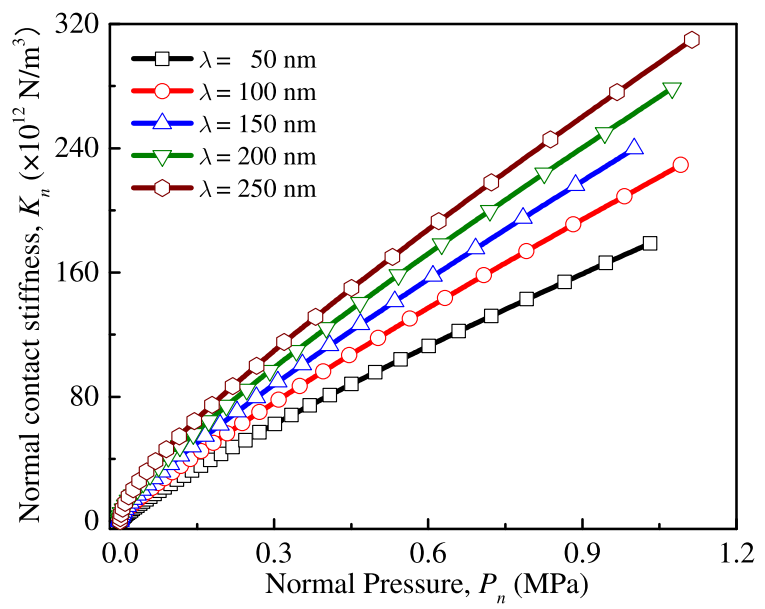

(b)

Fig. 8. Contact behavior of rough surfaces with different wavelengths $\lambda$ : (a) the relationships between the normal deformation $\delta_{n}$ and the normal pressure $P_{n}$ and (b) the relationships between the normal pressure $P_{n}$ and the normal contact stiffness $K_{n}$.

same $\delta_{n}$, the normal pressure decreases as either $R_{a}$ or $\psi$ increases, as reported in previous study [Zelasko et al., 2016]. According to Eq. (5), the areal plastic index $\psi$ exhibits a positive relationship with the standard deviation $R_{a}$, and here the ratio of $R_{q} / R_{a}=1.25$ Thomas, 1999; Kumar and Rao, 2012; Vitanov et al., 2014. Figure 9(b) shows the relationships between the normal pressure $P_{n}$ and the normal contact stiffness $K_{n}$ for rough surfaces with different roughness values $R_{a}$. For the same $P_{n}$, the normal contact stiffness decreases as either $R_{a}$ or $\psi$ increases. 


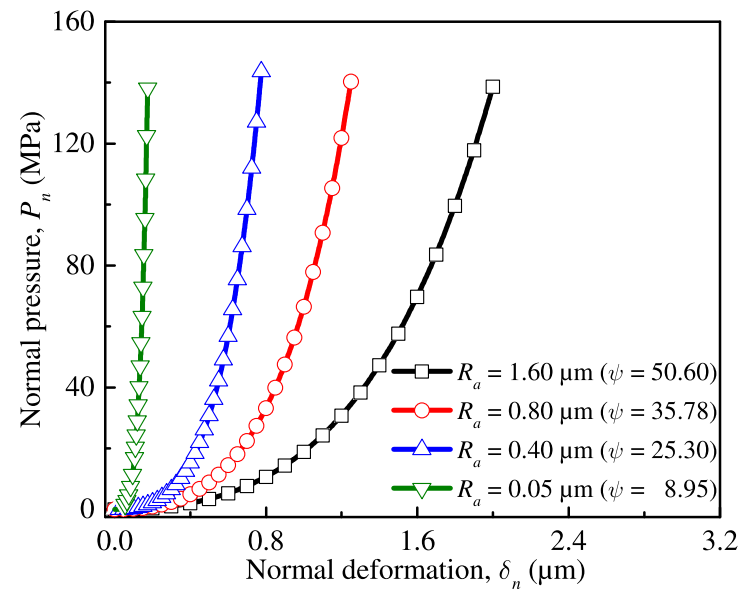

(a)

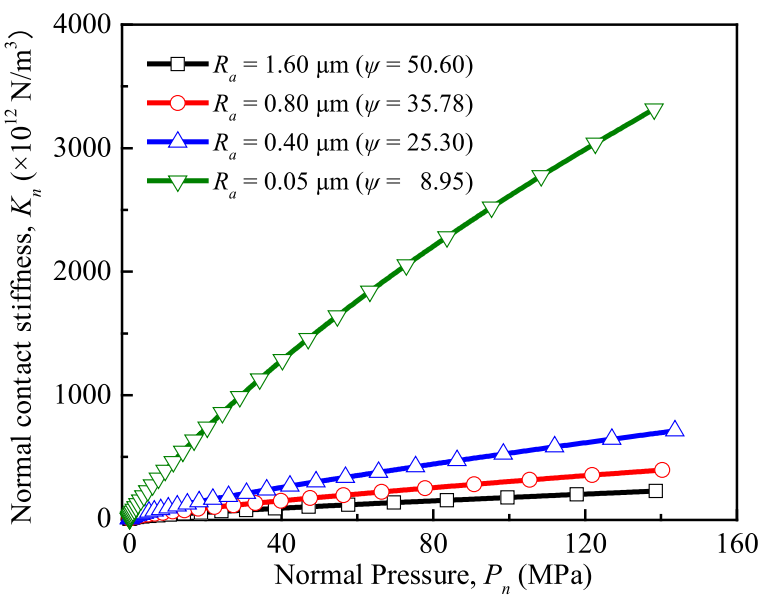

(b)

Fig. 9. Contact behavior of rough surfaces with different roughness $R_{a}$ : (a) the relationships between the normal deformation $\delta_{n}$ and the normal pressure $P_{n}$ and (b) the relationships between the normal pressure $P_{n}$ and the normal contact stiffness $K_{n}$.

\subsection{Contact behavior of rough surfaces with different standard deviations $\boldsymbol{R}_{q}$}

Standard deviation $R_{q}$ influences the normal pressure and the normal contact stiffness of a rough surface. A surface with a roughness of $R_{a} \leq 0.40 \mu \mathrm{m}$ has long-term stability, as required in most mechanical equipment. Five surfaces are selected, for which the roughness $R_{a}$ is $0.40 \mu \mathrm{m}$ and the ratios of $R_{q} / R_{a}$ are $0.00,1.00,1.10$, 1.25 , and 1.50 , respectively. Hence, the $R_{q}$ of these surfaces are $0.00,0.40,0.44$, 0.50 , and $0.60 \mu \mathrm{m}$, respectively. In addition, the five surfaces have identical mean 


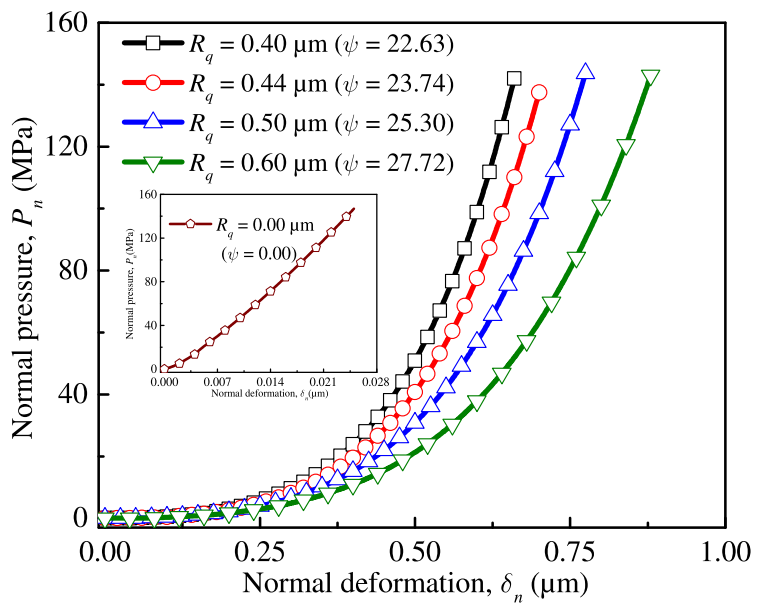

(a)

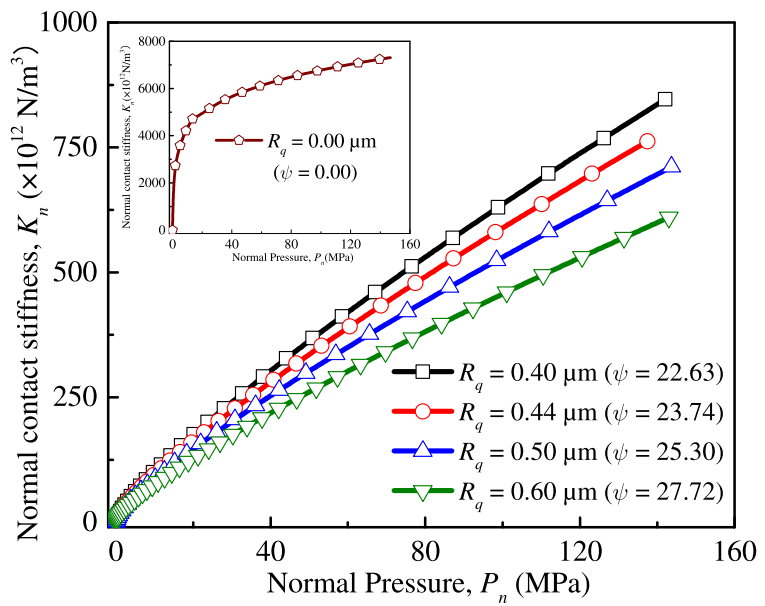

(b)

Fig. 10. Contact behavior of rough surfaces with different standard deviations $R_{q}$ : (a) the relationships between the normal deformation $\delta_{n}$ and the normal pressure $P_{n}$ and (b) the relationships between the normal pressure $P_{n}$ and the normal contact stiffness $K_{n}$.

curvature radius of asperity summits $R_{s . m}=21.08 \mu \mathrm{m}$ and the same surface density of asperities $\eta=40194 / \mathrm{mm}^{2}$ [hao et al., 2015].

Figure [10 (a) shows the relationships between the normal deformation $\delta_{n}$ and the normal pressure $P_{n}$ for rough surfaces with different standard deviations $R_{q}$. The normal pressure increases with the increase of the normal deformation. For the four surfaces with $R_{q}>0$, under the same $\delta_{n}$, the normal pressure decreases as either $R_{q}$ or $\psi$ increases, as reported in previous study ZZelasko et al., 2016]. For the surface with $R_{q}=0.00 \mu \mathrm{m}$, the relationship between the normal deformation 
and the normal pressure is consistent with the contact behavior between a single sinusoidal asperity and a rigid smooth flat.

Figure 10(b) shows the relationships between the normal pressure $P_{n}$ and the normal contact stiffness $K_{n}$ for rough surfaces with different standard deviations $R_{q}$. The normal contact stiffness increases with the increase of the normal pressure. For the four surfaces with $R_{q}>0$, under the same $P_{n}$, the normal contact stiffness decreases as either $R_{q}$ or $\psi$ increases. For the surface with $R_{q}=0.00 \mu \mathrm{m}$, the relationship of the normal pressure and the normal contact stiffness is consistent with the contact behavior between a single sinusoidal asperity and a rigid smooth flat.

\subsection{Comparison between the proposed model and the Greenwood-Williamson ( $G W)$ model}

Generally, two real contact surfaces in Zhao et al. [2015] are equivalent to a rough surface and a rigid flat in theoretical calculation. In this study, the morphology parameters of the equivalent surface are set as the parameters of the two real contact surfaces, as described below: the mean value of the asperity heights $\mu_{z}=0.31 \mu \mathrm{m}$, the standard deviation of the asperity heights $\sigma_{z}=6.20 \mu \mathrm{m}$, the mean curvature radius of asperity summits $R_{s, m}=21.08 \mu \mathrm{m}$, and the surface density of asperities $\eta=40194 / \mathrm{mm}^{2}$ Zhao et al., 2015]. The areal plasticity index of the 18CrMo4 steel interface $\psi=89.098$ is calculated by Eq. (5) . Asperity height $h$ follows the Gaussian distribution on rough surfaces. According to Poon and Bhushan' results [Poon and Bhushan, 1995], it is suggested that the sinusoidal shape is close to the profile from surface measurement. Thus, we use the sinusoidal shape to represent the $18 \mathrm{CrMo} 4$ steel surface. The shape ratio $\xi$ on sinusoidal rough surfaces can be calculated by Eq. (14)

$$
\xi=\frac{h}{\lambda}=\left(\frac{h}{2 \pi^{2} R_{s}}\right)^{1 / 2}=\left(\frac{h}{2 \pi^{2} R_{s, m}}\right)^{1 / 2} .
$$

The $18 \mathrm{CrMo} 4$ steel surface and the sinusoidal surface are connected by Eq. (14) which contains the actual morphology parameters (i.e., $h$ and $R_{s, m}$ ) and the shape ratio $\xi$ on the sinusoidal surface. These parameters are used in the proposed model to predict contact law of an $18 \mathrm{CrMo} 4$ steel interface.

The normal pressure $P_{n}$ and the normal contact stiffness $K_{n}$ calculated by the proposed model with the morphology parameters above are compared with the experimental results of Zhao et al. [2015]. In addition, the normal pressure and the normal contact stiffness obtained by the GW model [Greenwood and Williamson, 1966], which is based on the Hertz solution for spherical contact, are also plotted for comparison in Fig. 11. The results of the proposed model are closer to the experimental results than to the GW model, which overestimates both the real normal pressure and the real normal contact stiffness. The reason for such overestimation in the elastic GW model lies in that the areal plasticity index of the experimental 


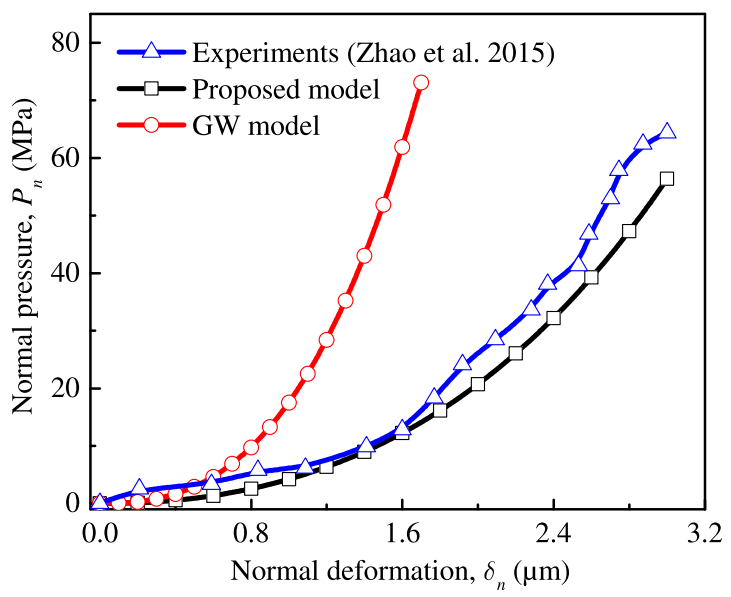

(a)

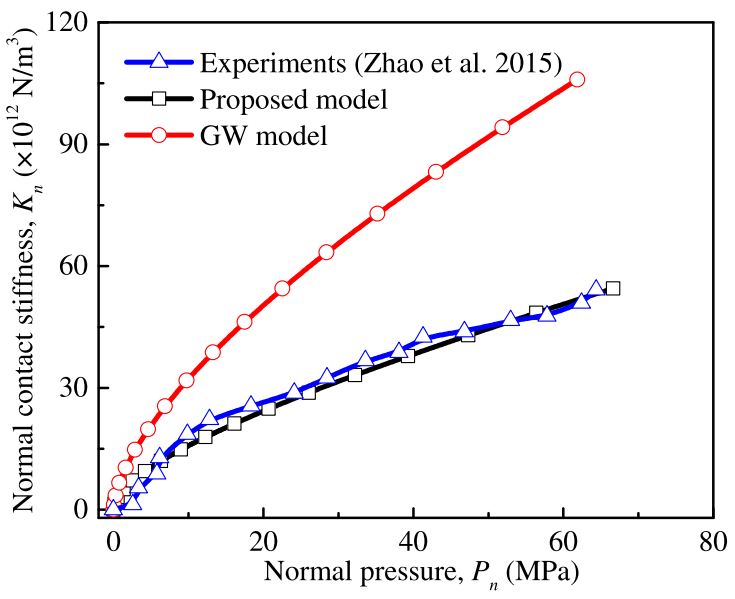

(b)

Fig. 11. Comparison of (a) the normal pressure $P_{n}$ and (b) the normal contact stiffness $K_{n}$ derived from different contact models and experimental results of $18 \mathrm{CrMo} 4$ steel interface by Zhao et al. 2015].

18CrMo4 interface $\psi(=89.098)$ is substantially greater than 1 , which means that the experimental interface mainly experiences plastic deformation. The proposed model is an elastic-plastic contact model with the consideration of plastic behavior of the $18 \mathrm{CrMo} 4$ steel material. In addition, sinusoidal contact better approximates the real situation than spherical contact. Hence, the proposed model agrees well with the experimental results of the contact behavior for an 18CrMo4 interface.

When the contacting surface is subjected to a high load, the interaction of asperities is obvious Chandrasekar et al., 2013]. The FE contact model of Gaussian 
surface established by Chandrasekar et al. shows that: (11) when $d_{s p} / R_{s, m} \in[0.20$, 1.50] and the contact deformation $\delta_{n} \geq 0.85 \sigma_{z}$, the interaction of asperities cannot be ignored; (2) when $d_{s p} / R_{s, m} \in[1.50, \infty]$, the interaction of asperities can be ignored Chandrasekar et al., 2013]. Where $d_{s p} / R_{s, m}$ is the ratio of asperity spacing $d_{s p}\left(=1 /(\eta)^{1 / 2}\right)$ to curvature radius of asperity summit $R_{s, m}$, and $\sigma_{z}$ is the standard deviation of the asperity heights. According to the results of Chandrasekar et al. [2013], the surface topography parameters of Zhao et al. 2015] can be calculated that the asperity spacing on $18 \mathrm{CrMo} 4$ steel surface is $d_{s p}=1 /(\eta)^{1 / 2}=1 /(40194)^{1 / 2}=4.99 \mu \mathrm{m}$ and $d_{s p} / R_{s, m}=0.24 \in[0.20,1.50]$. Therefore, the interaction of asperities is not significant when the deformation of $18 \mathrm{CrMo} 4$ steel surface $\delta_{n} \leq 0.85 \times 6.20=5.27 \mu \mathrm{m}$. The maximum deformation $\delta_{n}(=3.00 \mu \mathrm{m})$ of the proposed model in Sec. 3.5 is less than $5.27 \mu \mathrm{m}$. Therefore, the asperity on surface undergoes elastoplastic deformation and the interaction of asperities is negligible. The proposed model is elastoplastic contact model which ignores the interaction of asperities. Hence, the proposed model produces reasonable result, and it agrees well with the experimental results of Zhao et al. [2015].

\section{Conclusions}

Based on finite element analysis, this work presents a statistical elastic-plastic contact model between $18 \mathrm{CrMo} 4$ rough interfaces. The influences of the morphology parameters on the contact behavior are studied, and the proposed model is compared and validated with both the traditional elastic GW model and the experimental results of Zhao et al. 2015]. The following conclusions can be derived from this study:

- The proposed model agrees well with the experimental results, indicating the reliability of the statistical model with the consideration of the elastic-plastic contact behavior of single contact.

- For rough surfaces with identical roughness values $R_{a}$ and $R_{q}$, a smaller shape ratios $\xi$ and larger wavelengths $\lambda$ induce larger normal pressure and larger normal contact stiffness.

- For the same $\delta_{n}$ or $P_{n}$, the normal contact stiffness increases with the decrease of roughness $R_{a}$. For the same roughness $R_{a}$, the normal pressure and normal contact stiffness increase with the decrease of $R_{q}$.

\section{Acknowledgments}

This work was supported by the National Natural Science Foundation of China (Grant Nos. 11772334, 11572329, and 11672301) and by National Key R\&D Program of China (No. 2017YFA0204402). This work is also funded by Youth Innovation Promotion Association CAS (2018022) and by the Strategic Priority Research Program of the Chinese Academy of Sciences, Grant No. XDB22040501. 


\section{Appendix A. Effect of Width of Substrate on Sinusoidal Contact}

Yastrebov et al. 2011] studied the effect of width of substrate on contact response of force-deformation. The dimensions of the FE model are sinusoidal asperity width $\lambda=10 \mu \mathrm{m}$ and height $h=1 \mu \mathrm{m}$, thickness of substrate $H_{s}=72 \mu \mathrm{m}$, width of substrate is $L, 2 L$ and $4 L$, where $L=\lambda / 2$, as shown in Fig. A.1. The results of FE model show the width of substrate has a negligible effect in the range of $0-0.25 \mathrm{~h}$.

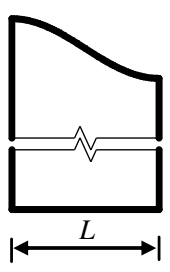

(a)

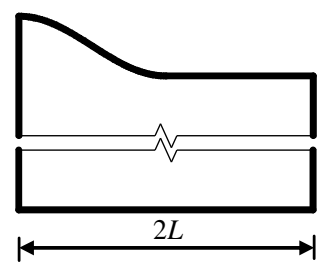

(b)

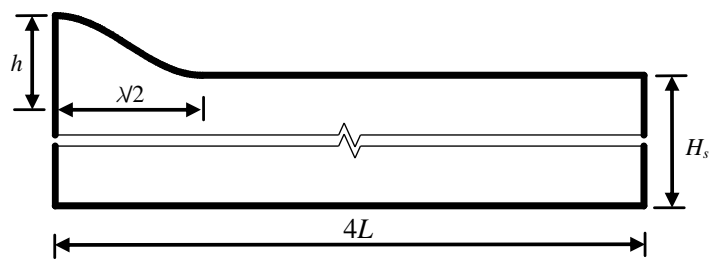

(c)

Fig. A.1. Different width of substrate.

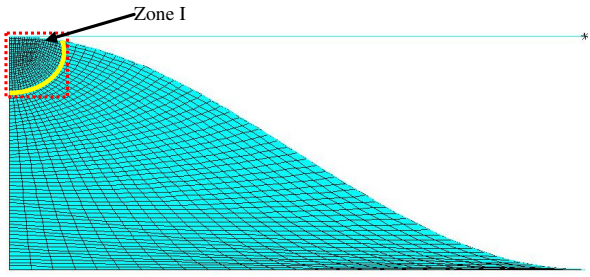

(a)

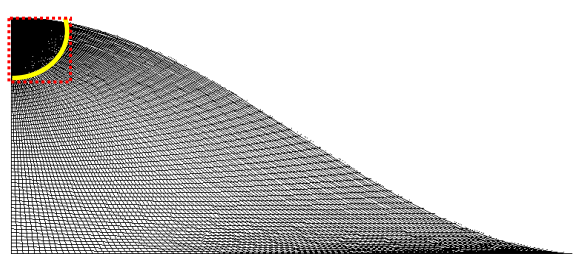

(c)

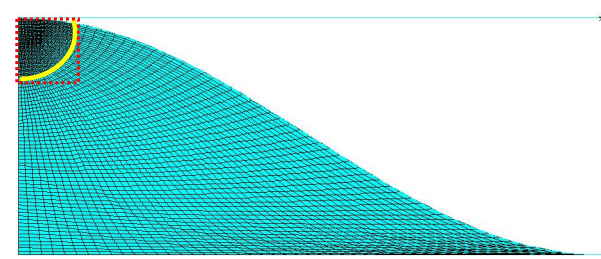

(b)

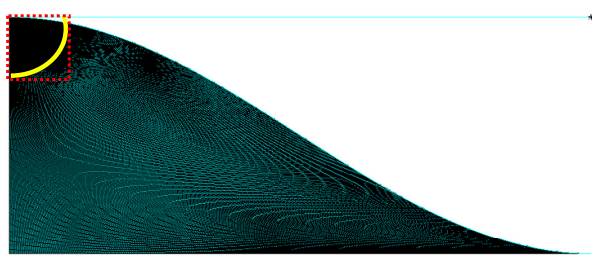

(d)

Fig. B.1. FEM of sinusoidal asperity (Shape ratio $\xi=0.20, \lambda=1 \mathrm{~mm}$ ), the mesh size of zone I: (a) $3 \lambda \times 10^{-3}$; (b) $\lambda \times 10^{-3}$; (c) $5 \lambda \times 10^{-4}$; (d) $\lambda \times 10^{-4}$. 
The influence of width of substrate should be considered under high load. However, in our work, small load or moderate load are applied to the rough surface. Therefore, the present model without considering width of substrate is reasonable.

\section{Appendix B. Mesh Sensitivity Analysis}

The FE model of a sinusoidal asperity with shape ratio $\xi(=h / \lambda)=0.20$ is used for mesh sensitivity analysis, where $\lambda=1 \mathrm{~mm}$. The contact tip is the focal monitoring area with refined mesh, the mesh size of zone $\mathrm{I}$ is $3 \lambda \times 10^{-3}, \lambda \times 10^{-3}, 5 \lambda \times 10^{-4}$ and $\lambda \times 10^{-4}$, respectively, as shown in Fig. B.1. The mesh size of other areas is $3 \lambda \times 10^{-3}$. Figure B.2 shows the relationship between the normal deformation $\delta_{n}$

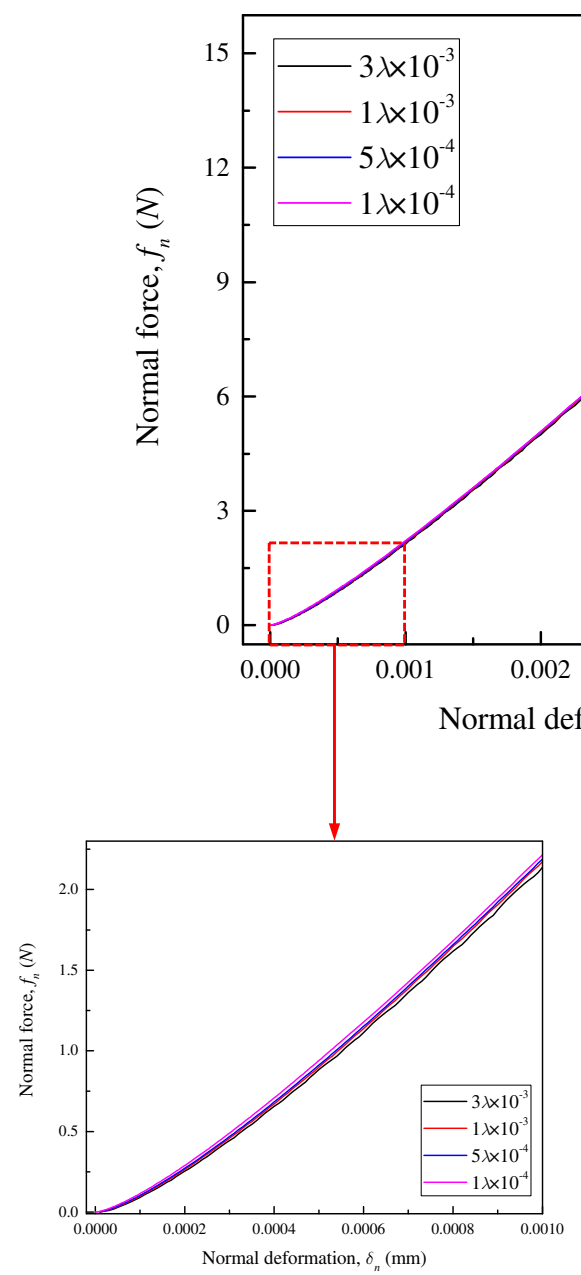

(a)

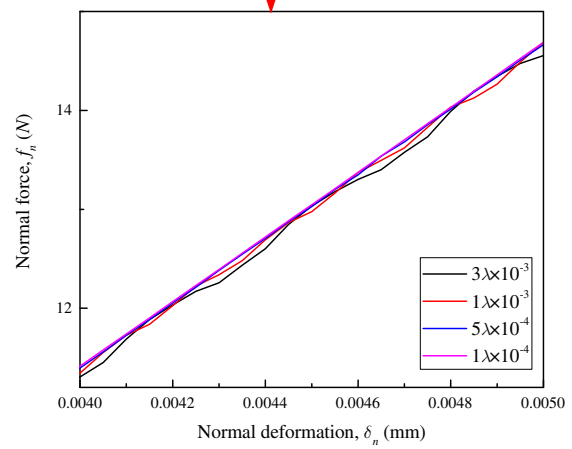

(b)

Fig. B.2. The relationship between the normal force $f_{n}$ and the normal deformation $\delta_{n}$. 
and the normal force $f_{n}$ with different mesh size. The mesh size has little effect on the normal force-the normal deformation relationship. The force-deformation curve in the penetration range of $0-0.001 \mathrm{~mm}$ and $0.004-0.005 \mathrm{~mm}$ are enlarged, as shown in Figs. B.2(a) and B.2(b), respectively. The force-deformation curves of mesh size $\lambda \times 10^{-4}$ and $5 \lambda \times 10^{-4}$ are smoother and closer than that of mesh size of $\lambda \times 10^{-3}$ and $3 \lambda \times 10^{-3}$. Therefore, the mesh size of zone $\mathrm{I}$ is meshed by $\lambda \times 10^{-4}$.

\section{References}

Boscolo, M. and Banerjee, J. R. [2012] "Dynamic stiffness formulation for composite Mindlin plates for exact modal analysis of structures. Part II: results and applications," Computers \& Structures 96-97, 74-83.

Chandrasekar, S., Eriten, M. and Polycarpou, A. A. [2013] "An improved model of asperity interaction in normal contact of rough surfaces," Journal of Applied Mechanics 80, 011025 .

Ciavarella, M., Demelio, G., Barber, J. R. and Jang, Y. H. [2000] "Linear elastic contact of the weierstrass profile," Proceedings of the Royal Society of London. Series A: Mathematical, Physical and Engineering Sciences 456, 387-405.

Du, J., Chen, X. F., Liang, X. D., Zhang, G. Y., He, L. R., Zhan, Q. Y., Feng, X. Q., Chien, S. and Yang, C. [2011] "Integrin activation and internalization on soft ECM as a mechanism of induction of stem cell differentiation by ECM elasticity," Proceedings of the National Academy of Sciences of the United States of America 108(23), 94669471.

Gao, Y. F., Bower, A. F., Kim, K.-S., Lev, L. and Cheng, Y. T. [2006] "The behavior of an elastic-perfectly plastic sinusoidal surface under contact loading," Wear 261, $145-154$.

Giraudeau, A., Pierron, F. and Guo, B. Q. [2010] "An alternative to modal analysis for material stiffness and damping identification from vibrating plates," Journal of Sound and Vibration 329(10), 1653-1672.

Greenwood, J. A. and Williamson, J. B. P. [1966] "Contact of nominally flat surfaces," Proceedings The Royal of Society. Series A, Mathematical Physical and Engineering Sciences 295, 300-319.

Greenwood, J. A. and Tripp, J. H. [1970] "The contact of two nominally flat rough surfaces," Procceedings of the Institution of Mechanical Engineers 185(71), 625-633.

Hertz, H. [1882] "On the contact of elastic solids," Journal Fur Die Reine Und Angewandte Mathematik 92, 156-171.

Hisakado, T. [1975] "Surface roughness and deformation of contact asperities between a rough and a flat surface," Wear 35, 53-61.

Jackson, R. L. and Streator, J. L. [2006] "A multiscale model for contact between rough surfaces," Wear 261(11-12), 1337-1347.

Jackson, R. L., Krithivasan, V. and Wilson, W. E. [2008] "The pressure to cause complete contact between elastic-plastic sinusoidal surfaces," Proceedings of the Institution of Mechanical Engineers, Part J-Journal of Engineering Tribology 222, 857-863.

Jamari, J. and Schipper, D. J. [2006] "An elastic-plastic contact model of ellipsoid bodies," Tribology Letters 21(3), 262-271.

Johnson, K. L. [1985] Contact Mechanics (Cambridge University Press, Cambridge).

Johnson, K. L., Greenwood, J. A. and Higginson, J. G. [1985] "The contact of elastic regular wavy surfaces," International Journal of Mechanical Sciences 27(6), 383-396. 
Kartal, M. E., Mulvihill, D. M., Nowell, D. and Hills, D. A. [2011] "Measurements of pressure and area dependent tangential contact stiffness between rough surfaces using digital image correlation," Tribology International 44(10), 1188-1198.

Kogut, L. and Etsion, I. [2002] "Elastic-plastic contact analysis of a sphere and a rigid flat," Journal of Applied Mechanics Transactions of The ASME 69, 657-662.

Kumar, B. R. and Rao, T. S. [2012] "AFM studies on surface morphology, topography and texture of nanostructured zinc aluminum oxide thin films," Digest Journal of Nanomaterials and Biostructures 7(4), 1881-1889.

Li, S., Yao, Q. Z., Li, Q. Y., Feng, X. Q. and Gao, H. J. [2018] "Contact stiffness of regularly patterned multi-asperity interfaces," Journal of the Mechanics and Physics of Solids 111, 277-289.

Liu, M. [2014] "Finite element analysis of large contact deformation of an elastic-plastic sinusoidal asperity and a rigid flat," International Journal of Solids and Structures 51, 3642-3652.

Liu, M. and Proudhon, H. [2014] "Finite element analysis of frictionless contact between a sinusoidal asperity and a rigid plane: Elastic and initially plastic deformations," Mechanics of Materials 77, 125-141.

Liu, M. and Proudhon, H. [2016] "Finite element analysis of contact deformation regimes of an elastic-power plastic hardening sinusoidal asperity," Mechanics of Materials 103, 78-86.

Liu, Z. Q., Shi, J. P., Wang, F. S. and Yue, Z. F. [2015] "Normal contact stiffness of the elliptic area between two asperities," Acta Mechanica Solida Sinica 28(1), 33-39.

Lu, P., Yang, L. L. and Wang, G. F. [2017] "Studies of low-loading micro-slip contacts on rough surfaces with GW model," International Journal of Applied Mechanics 9(4), 1750049.

Lu, X. Q., Huang, F. Z., Zhao, B. and Keer, L. M. [2018] "Contact behaviors of coated asperity with power-law hardening elastic-plastic substrate during loading and unloading process," International Journal of Applied Mechanics 10(3), 1850034 .

Mandelbrot, B. B. [1983] The Fractal Geometry of Nature (Macmillan, London).

Pan, W. J., Li, X. P., Wang, L. L., Guo, N. and Mu, J. X. [2017] "A normal contact stiffness fractal prediction model of dry-friction rough surface and experimental verification," European Journal of Mechanics A/Solids 66, 94-102.

Poon, C. Y. and Bhushan, B. [1995] "Comparison of surface roughness measurements by stylus profiler, AFM and non-contact optical profiler," Wear 190, 76-88.

Ramberg, W. and Osgood, W. R. [1943] "Description of stress-strain curves by three parameters," National Advisory Committee for Aeronautics Technical Note No. 902, pp. 1-28.

Saha, S. and Jackson, R. L. [2016] "Elastic-plastic axisymmetric sinusoidal surface asperity contact," in IEEE 62nd Holm Conference on Electrical Contacts, Clearwater Beach, Florida, pp. 17-24.

Saha, S., Xu, Y. and Jackson, R. L. [2016] "Perfectly elastic axisymmetric sinusoidal surface asperity contact," Journal of Tribology-Transactions of The ASME 138(3), 031401.

Shah, S., Krithivasan, V. and Jackson, R. L. [2011] "An electro-mechanical contact analysis of a three-dimensional sinusoidal surface against a rigid flat," Wear 270, 914921.

Tabor, D. [1951] The Hardness of Metals (Oxford University Press, Oxford).

Thomas, T. R. [1999] Rough Surfaces (Imperial College Press, London).

Timsit, R. S. [2006] "Electrical conduction through small contact spots," IEEE Transactions on Components and Packaging Technologies 29, 727-734. 
Vitanov, P., Harizanova, A., Ivanova, T. and Dikov, H. [2014] "Low-temperature deposition of ultrathin $\mathrm{SiO}_{2}$ films on Si substrates," Journal of Physics: Conference Series 514, 012010.

Wang, R. Q., Zhu, L. D. and Zhu, C. X. [2017] "Research on fractal model of normal contact stiffness for mechanical joint considering asperity interaction," International Journal of Mechanical Sciences 134, 357-369.

Wang, X. Z., Xu, Y. and Jackson, R. L. [2017] "Elastic sinusoidal wavy surface contact under full stick conditions," Tribology Letters 65, 156-169.

Westergaard, H. M. [1939] "Bearing pressure and cracks," Journal of Applied Mechanics 38, 49-53.

Wilson, W. E., Angadi, S. V. and Jackson, R. L. [2010] "Surface separation and contact resistance considering sinusoidal elastic-plastic multi-scale rough surface contact," Wear 268, 190-201.

Xiao, H. F. and Sun, Y. Y. [2018] "An improved virtual material based acoustic model for contact stiffness measurement of rough interface using ultrasound technique," International Journal of Solids and Structures 155, 240-247.

Yastrebov, V. A., Durand, J., Proudhon, H. and Cailletaud, G. [2011] "Rough surface contact analysis by means of the finite element method and of a new reduced model," Comptes Rendus Mecanique 339(7-8), 473-490.

$\mathrm{Yu}$, N. and Polycarpou, A. A. [2004] "Combining and contacting of two rough surfaces with asymmetric distribution of asperity heights," Journal of Tribology 126, 225-232.

Zelasko, W., Pawlus, P., Dzierwa, A. and Prucnal, S. [2016] "Experimental investigation of plastic contact between a rough steel surface and a flat sintered carbide surface," Tribology International 100, 141-151.

Zhao, B., Zhang, S., Wang, P. and Hai, Y. [2015] "Loading-unloading normal stiffness model for power-law hardening surfaces considering actual surface topography," Tribology International 90, 332-342. 